BMJ Open

Sport \&

Exercise

Medicine

\section{Predictive ability of a comprehensive incremental test in mountain bike marathon}

To cite: Ahrend M-D, Schneeweiss P, Martus P, et al. Predictive ability of a comprehensive incremental test in mountain bike marathon. BMJ Open Sport \& Exercise Medicine 2018;0:e000293. doi:10.1136/ bmjsem-2017-000293

Accepted 20 December 2017

\section{Check for updates}

${ }^{1}$ Department of Sports Medicine, Medical Clinic, University of Tuebingen, Tuebingen, Germany ${ }^{2}$ Institute for Clinical Epidemiology and Applied Biometry, University Hospital Tuebingen, Tuebingen, Germany

Correspondence to Dr. Marc-Daniel Ahrend; marc@ ahrend.de

\section{ABSTRACT}

Objectives Traditional performance tests in mountain bike marathon (XCM) primarily quantify aerobic metabolism and may not describe the relevant capacities in XCM. We aimed to validate a comprehensive test protocol quantifying its intermittent demands.

Methods Forty-nine athletes (38.8 \pm 9.1 years; 38 male; 11 female) performed a laboratory performance test, including an incremental test, to determine individual anaerobic threshold (IAT), peak power output (PPO) and three maximal efforts (10 s all-out sprint, 1 min maximal effort and 5 min maximal effort). Within 2 weeks, the athletes participated in one of three XCM races $(n=15$, $n=9$ and $n=25$ ). Correlations between test variables and race times were calculated separately. In addition, multiple regression models of the predictive value of laboratory outcomes were calculated for race 3 and across all races (z-transformed data).

Results All variables were correlated with race times 1 , 2 and 3 : 10 s all-out sprint $(r=-0.72 ; r=-0.59 ; r=-0.61)$, 1 min maximal effort $(r=-0.85 ; r=-0.84 ; r=-0.82)$, 5 min maximal effort $(r=-0.57 ; r=-0.85 ; r=-0.76)$, PPO $(r=-0.77 ; r=-0.73 ; r=-0.76)$ and IAT $(r=-0.71 ; r=-0.67$; $r=-0.68)$. The best-fitting multiple regression models for race $3\left(r^{2}=0.868\right)$ and across all races $\left(r^{2}=0.757\right)$ comprised 1 min maximal effort, IAT and body weight. Conclusion Aerobic and intermittent variables correlated least strongly with race times. Their use in a multiple regression model confirmed additional explanatory power to predict XCM performance. These findings underline the usefulness of the comprehensive incremental test to predict performance in that sport more precisely.

\section{INTRODUCTION}

Mountain bike marathon (XCM) is one of the main disciplines in mountain biking (MTB) and is characterised as high-intensity intermittent activity because of its multitude of climbs and downhill sections. ${ }^{1-4}$ The durations and track profiles of XCM races can vary from $90 \mathrm{~min}$ up to 6 hours or from $60 \mathrm{~km}$ to $160 \mathrm{~km} .{ }^{5}$ Therefore, exercise intensities may differ depending on race characteristics. ${ }^{67}$

XCM has become increasingly popular over the last years as a recreational and competitive sport. ${ }^{45}$ With increasing popularity, also the need for a valid performance test has become

\section{What are the new findings?}

- The comprehensive incremental test showed sufficient validity and comprised traditional parameters (individual anaerobic threshold (IAT) and peak power output) as well as additional maximal efforts with durations of $10 \mathrm{~s}, 1 \mathrm{~min}$ and $5 \mathrm{~min}$.

- The power output of a 1 min maximal effort following an incremental test and a $10 \mathrm{~s}$ all-out sprint was the most valuable single variable to predict mountain bike marathon race performance.

- In multiple regression models, up to $86.8 \%$ variance of the race time of one race and $75.7 \%$ variance of race times across three races can be explained using the power output in a 1 min maximal effort, the IAT and the body weight.

more important. Performance tests are relevant to facilitate training and predict race performance in competitive and recreational athletes. ${ }^{9}$ To ensure this, a performance test should cover the physiological demands of the sport comprehensively ${ }^{10}$ and predict race performance for mountain bike races sufficiently.

The traditional approach to predicting race performance is an incrementally increasing laboratory test on a bicycle ergometer. Although previous studies ${ }^{11-13}$ have indicated that incremental tests predict mountain bike race performance sufficiently, its predictive ability to explain the variance of race performance differ from $44 \%$ to $92 \%$ between the studies. ${ }^{11-13}$ Therefore, Prins et $a l^{12}$ described the need for a better tailored laboratory test design and suggested a shift towards less traditional laboratory tests that consider the demands of MTB. ${ }^{21214}$

Therefore, we conducted a pilot study to validate a comprehensive test battery in 10 athletes to find laboratory parameters that have the potential to improve the prediction of mountain bike performance. The test battery comprised a traditional incremental test, maximal strength testing and a 
laboratory-simulated time trial consisting of maximal efforts with durations of $10 \mathrm{~s}, 1 \mathrm{~min}$ and $5 \mathrm{~min}$. The chosen durations of maximal efforts are considered to be typical in MTB and are often used for training purposes. ${ }^{1415}$ The results of the pilot study showed that power outputs of maximal efforts with durations of 1 and 5 min have similar correlations with race performance compared with traditional parameters of an incremental test. These findings underlined that traditional aerobic parameters of an incremental test, as well as power output during short high-intensive efforts, should be considered when analysing mountain bike performance.

However, this pilot study as well as other previously published studies ${ }^{1} 4111316-19$ used small sample sizes and analysed the race prediction of the mountain bike discipline Olympic cross-country (XCO), which is more intense and shorter in duration. ${ }^{4} 7$

Therefore, this study aimed to determine the predictive ability as part of the criterion validity of a comprehensive incremental test. The performance test was designed on the basis of the results of a pilot study ${ }^{20}$ and comprised the traditional parameters of an incremental test as well as maximal efforts. We hypothesised that the predictive ability of the comprehensive incremental test outperformed the predictive ability of the traditional incremental test.

\section{METHODS}

\section{Study design}

This is a validation study that was conducted during the athletes' competitive phase of their season. All data were recorded within 5 weeks to minimise seasonal changes in the athletes' performance levels. The study was divided into three examination sections. Participants of each section performed a laboratory performance test and subsequently competed in an official national XCM

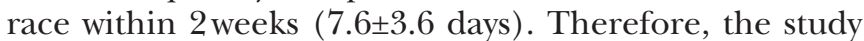
comprised three cohorts with three different XCM races (see figure 1).

\section{Participants}

Forty-nine participants (age: $38.8 \pm 9.1$ years) with XCM experience of $10.6 \pm 7.1$ years participated in this study. They were mostly classified as trained athletes (performance level 3) following the absolute peak power output (PPO) $(329 \pm 49 \mathrm{~W})$ criteria of De Pauw et al. ${ }^{21}$ Further biometric and training data on the participants are shown in table 1 .

Sample size estimation was based on the results of a pilot study. ${ }^{20}$ Because of the short duration of the study, the largest possible sample size to include in the study was 50 . According to the results of the pilot study, ${ }^{20} \mathrm{a}$ sample of 50 participants would have explained $r^{2}=0.64$

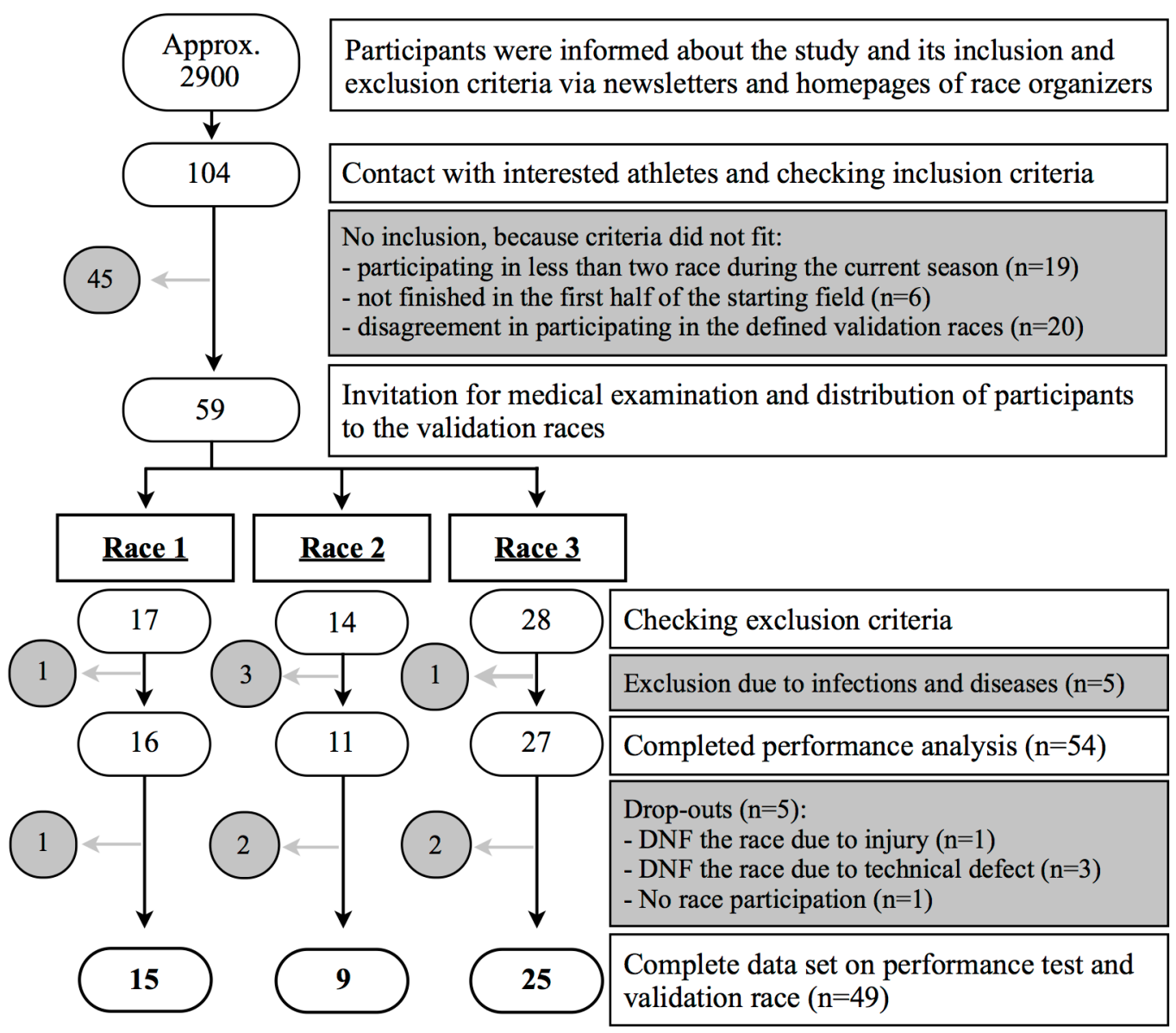

Figure 1 Study flow chart. DNF, did not finish the race. 
Table 1 Athletes' characteristics and values (absolute and scaled by body weight) of laboratory variables (mean \pm SD)

\begin{tabular}{|c|c|c|c|c|}
\hline & $\begin{array}{l}\text { All athletes } \\
n=49\end{array}$ & $\begin{array}{l}\text { Race } 1 \\
n=15\end{array}$ & $\begin{array}{l}\text { Race } 2 \\
n=9\end{array}$ & $\begin{array}{l}\text { Race } 3 \\
n=25\end{array}$ \\
\hline Gender (male/female) & $38 / 11$ & $11 / 4$ & $8 / 1$ & $19 / 6$ \\
\hline Age (years) & $38.8 \pm 9.1$ & $38.2 \pm 7.9$ & $43.4 \pm 7.9$ & $37.6 \pm 9.9$ \\
\hline Height (cm) & $176.2 \pm 7.5$ & $175.7 \pm 8.4$ & $179.7 \pm 5.4$ & $175.3 \pm 7.5$ \\
\hline Body weight (kg) & $72.2 \pm 10.4$ & $72.6 \pm 10.3$ & $78.3 \pm 13.2$ & $69.9 \pm 8.9$ \\
\hline \multicolumn{5}{|l|}{ Cycling status } \\
\hline Races per year & $5.9 \pm 4.1$ & $5.2 \pm 3.2$ & $8.0 \pm 6.1$ & $5.5 \pm 3.5$ \\
\hline XCM experience (years) & $10.6 \pm 7.1$ & $10.9 \pm 5.2$ & $14.2 \pm 8.9$ & $9.1 \pm 7.2$ \\
\hline Training frequency/week & $4.4 \pm 1.6$ & $4.0 \pm 1.3$ & $5.0 . \pm 2.8$ & $4.4 \pm 1.1$ \\
\hline Training hours/week & $10.2 \pm 3.1$ & $10.3 \pm 3.2$ & $9.5 \pm 3.3$ & $10.5 \pm 3.1$ \\
\hline Training distance (km/week) & $218 \pm 129$ & $258 \pm 195$ & $175 \pm 70$ & $210 \pm 84$ \\
\hline \multicolumn{5}{|l|}{ Absolute values } \\
\hline PPO (W) & $329.1 \pm 49.4$ & $319.6 \pm 58.1$ & $336.3 \pm 21.4$ & $332.3 \pm 51.6$ \\
\hline 10 s all-out sprint $(\mathrm{W})$ & $764.3 \pm 158.7$ & $763.5 \pm 181.8$ & $783.2 \pm 83.4$ & $757.9 \pm 169.2$ \\
\hline 1 min maximal effort (W) & $439.8 \pm 89.1$ & $415.9 \pm 100.9$ & $458.5 \pm 63.0$ & $447.3 \pm 90.3$ \\
\hline 5 min maximal effort (W) & $271.2 \pm 49.3$ & $256.5 \pm 45.3$ & $279.4 \pm 33.2$ & $277.1 \pm 55.7$ \\
\hline \multicolumn{5}{|l|}{ Values scaled by body weight } \\
\hline IAT (W/kg) & $3.08 \pm 0.40$ & $2.95 \pm 0.34$ & $3.06 \pm 0.51$ & $3.17 \pm 0.38$ \\
\hline PPO (W/kg) & $4.58 \pm 0.51$ & $4.39 \pm 0.45$ & $4.40 \pm 0.65$ & $4.76 \pm 0.45$ \\
\hline 10 s all-out sprint (W/kg) & $10.57 \pm 1.68$ & $10.45 \pm 1.78$ & $10.17 \pm 1.57$ & $10.79 \pm 1.69$ \\
\hline 1 min maximal effort $(\mathrm{W} / \mathrm{kg})$ & $6.12 \pm 0.90$ & $5.69 \pm 0.93$ & $6.03 \pm 0.81$ & $6.41 \pm 0.84$ \\
\hline 5 min maximal effort $(\mathrm{W} / \mathrm{kg})$ & $3.78 \pm 0.52$ & $3.54 \pm 0.43$ & $3.68 \pm 0.46$ & $3.95 \pm 0.54$ \\
\hline
\end{tabular}

IAT, individual anaerobic threshold; PPO, peak power output; XCM, mountain bike marathon.

(CI 0.45 to 0.77 ), which was considered to be sufficient for validating the performance test. With an assumed dropout rate of $10 \%, 55$ participants should have been allocated to the study. Figure 1 displays the study flow chart with inclusion and exclusion criteria.

\section{Laboratory performance test}

After the medical examination was conducted and anthropometric measures were taken, the athletes underwent the laboratory performance test on an SRM Ergometer (SRM, Schoberer Rad Messtechnik, Jülich, Germany), which was individually adjusted (seat post, handle bar, pedal system). The heart rate was continuously recorded by a Polar heart rate monitor not-coded-model (Polar Electro, Kempele, Finland). The performance test started with an incremental exercise test at an entry load of $80 \mathrm{~W}$. The resistance was increased by $40 \mathrm{~W}$ every $3 \mathrm{~min}$ until exhaustion. During the incremental test, the athletes were advised to maintain a cadence between 70 and 100 revolutions per minute (rpm). The test was finished voluntarily by the athletes or if they could not maintain a cadence higher than $70 \mathrm{rpm}$. Lactate was analysed (Biosen S-Line, EKF, Cardiff, UK) by collecting capillary blood samples $(20 \mu \mathrm{L})$ from the right earlobe during the last $20 \mathrm{~s}$ of each stage. The individual anaerobic threshold (IAT (W)) was determined by Dickhuth $e$ t $a l^{22}$ and Roecker $e t a l^{23}$ and was calculated as a lactate concentration of $1.0 \mathrm{mmol} / \mathrm{L}$ above the lactate threshold, which is defined as the lowest value of the lactate to performance ratio and describes the onset of lactate increase.

After exhaustion and reaching the PPO, the athletes continued pedalling and proceeded with the performance test (see figure 2). It comprised a $7 \mathrm{~min}$ recovery period followed by a $10 \mathrm{~s}$ all-out sprint, another $3 \mathrm{~min}$ recovery period followed by a $1 \mathrm{~min}$ maximal effort, another $7 \mathrm{~min}$ recovery period followed by a $5 \mathrm{~min}$ maximal effort, and a final 5 min recovery period. The cadence for the three maximal efforts was fixed at $100 \mathrm{rpm}$ using the isokinetic function of the ergometer. Using the isokinetic function, the athletes pedalled up to the fixed cadence without resistance, while the resistance was automatically adjusted to the athletes' workload. ${ }^{24}$ During recovery periods, the athletes pedalled at a power output of 100 $\mathrm{W}$ and a free chosen cadence between 70 and $100 \mathrm{rpm}$. The athletes could choose between seated and standing position and were instructed to perform each interval with maximum effort. Power was recorded every $0.5 \mathrm{~s}$, and the mean power outputs for the $10 \mathrm{~s}$ all-out sprint, the 1 min maximal effort and the 5 min maximal effort were calculated, respectively.

\section{XCM races}

To provide evidence of the validity of laboratory variables, the athletes participated in one of three official national 


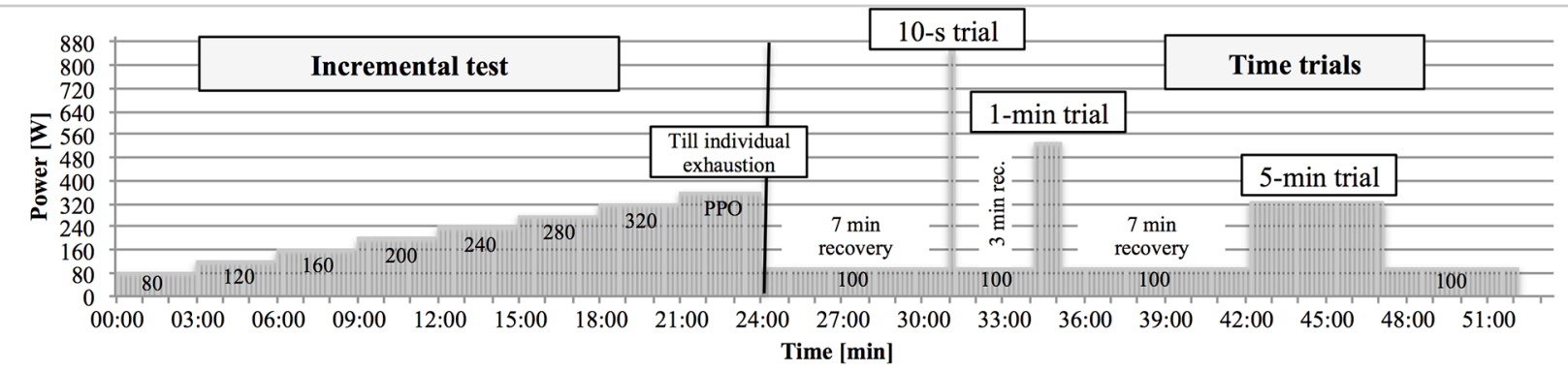

Figure 2 Test protocol with incremental test and maximal efforts.

XCM races with different race characteristics (see figure 3). Athletes participating in the study finished race 1 (distance: $90 \mathrm{~km}$, vertical ascent: $2100 \mathrm{~m}$, overall participants: 243) after 04:23:45 \pm 00:32:30 (from 03:36:51 to 05:37:59). Race 2 ( $56 \mathrm{~km}, 950 \mathrm{~m}$, overall participants: 450 ) was the first stage of the three stages of a stage race and lasted 02:23:35 $\pm 00: 12: 49$ (from 02:05:14 to 02:47:57). Race $3(83 \mathrm{~km}, 1700 \mathrm{~m}$, overall participants: 571) was finished after 03:12:42 $\pm 00: 16: 48$ (range: 02:39:23 to 03:43:13).

The athletes were encouraged to complete the race as fast as possible. Because the start position might influence race time, ${ }^{1125}$ the athletes were instructed to start in the first rows of the starting field. The official race time supplied by the race organiser was used for all the analyses. The athletes were asked through an online survey about their start position, possible race disturbances and interruptions, such as falls or technical problems. Athletes with technical problems or injuries during the races were excluded.

\section{Statistical analysis}

Race times were used as criterion variables for race-specific validation of the laboratory variables. For the overall analysis, race times were z-transformed (z-time) within races to account for differences in race characteristics (average race time and also variability of times). According to the Shapiro-Wilk test, the assumption of normally distributed data could be sustained for all data. To present the predictive value of laboratory variables and differences between races, laboratory variables were scaled by body weight (power divided by body weight $(\mathrm{W} / \mathrm{kg}),{ }^{46}$ and Pearson's correlation coefficient $r$ was used for the quantitative measure of the correlation between each laboratory variable and race times $1(\mathrm{n}=15), 2(\mathrm{n}=9), 3(\mathrm{n}=25)$ and standardised race time $(n=49)$. The coefficient was interpreted using a scale proposed by Hopkins. ${ }^{27}$

To test the influence of laboratory variables (absolute values (W) of IAT, PPO, 10s all-out sprint, 1 min maximal effort and 5 min maximal effort) and anthropometric variables (body weight, body mass index, body height, body fat and age) on race performance, backward multiple stepwise regressions with their adjusted $r^{2}$ were calculated for race time 3 and standardised z-time for all races, separately. Multiple regressions for race times 1 and 2 were not calculated because of the small subsample sizes. A value of $\mathrm{P}=0.10$ was selected as the probability of leaving and a value of $\mathrm{P}=0.05$ was selected as the probability of entering the stepwise multiple regressions. Leaving-one-out cross-validation was used to estimate the accuracy of the multiple regression models in practice. The study data were analysed with JMP (SAS Institute, JMP V.13.0.0, Cary, North Carolina, USA) at a level of significance of $\alpha=0.05$.

\section{RESULTS}

Table 1 shows the values of laboratory variables. All traditionally used parameters as well as the maximal efforts showed large to very large correlation with race times 1,2 and 3 (see figure 4). The IAT showed large to very large correlations $(\mathrm{r}=-0.67$ to -0.71$)$ and PPO showed very large correlations ( $\mathrm{r}=-0.73$ to -0.77$)$. The strongest correlations were found for $1 \mathrm{~min}$ maximal effort $(\mathrm{r}=-0.82$ to -0.85$)$.

Table 2 shows the backward stepwise regression between the absolute laboratory and anthropometric variables and race time 3 and z-time. The same predictors were included in both analyses (IAT, body weight and 1 min maximal effort). They explained the variance of race times with $86.8 \%$ (race time 3 ) and $75.7 \%$ (z-time).
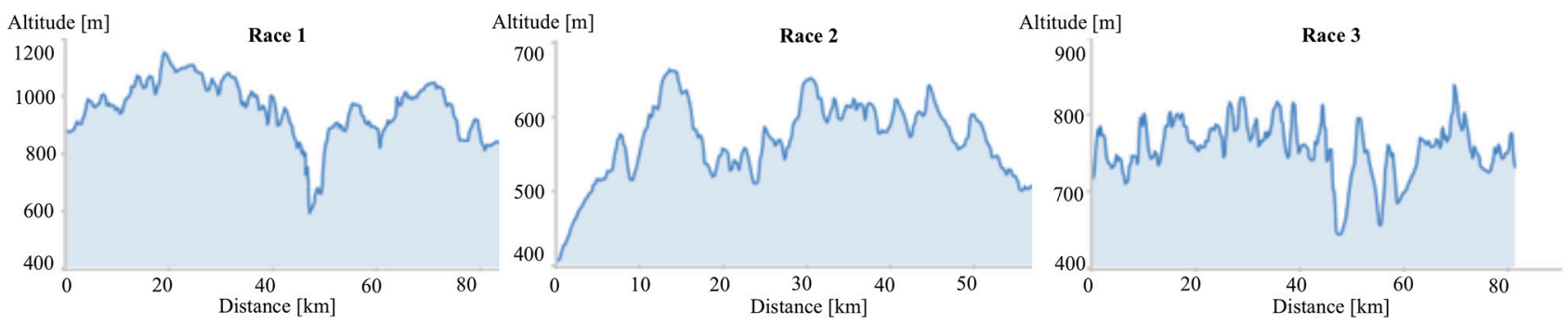

Figure 3 Course profiles of race 1, 2 and 3 . 


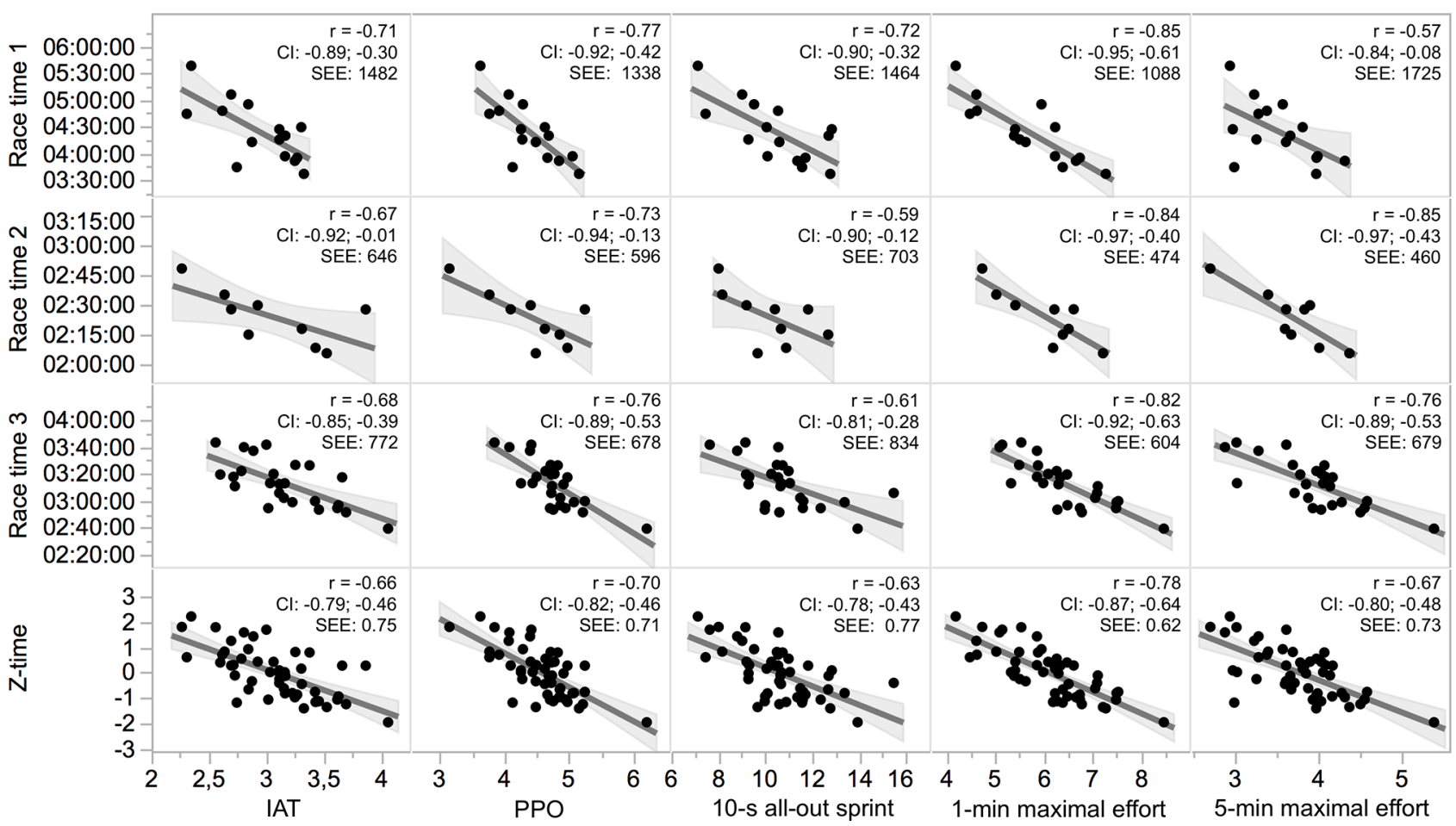

Figure 4 Linear regression models $(95 \% \mathrm{Cl}$; standard error of estimate (SEE, s)) of laboratory variables (values scaled by body weight (W/kg)) and race times 1, 2 and 3 and across all races (z-time). IAT, individual anaerobic threshold; PPO, peak power output.

In both models, the 1 min maximal effort had the highest predictive value.

\section{DISCUSSION}

In this study, we analysed the predictive ability of a laboratory test for race performance in XCM. The test comprised a traditional incremental cycle ergometer test and maximal efforts with durations typical for MTB. ${ }^{14} 15$ In comparison with the parameters of the traditional incremental test (IAT and PPO), the $1 \mathrm{~min}$ maximal effort showed stronger correlations with mountain bike races. Using these three variables in a multiple regression model, race performance can be predicted more precisely. The results demonstrate the importance of the combined role of aerobic (IAT) and high-intensity physiological demands in XCM. Referring to the aims of the study, it can be postulated that the comprehensive incremental test outperforms the traditional incremental test with regard to the predictive ability.

\section{Comparison between the parameters of the comprehensive incremental test}

The traditional laboratory variables of the incremental test showed large to very large correlations and could partly explain the race performance of the different validation XCM races with values between $45 \%$ and $50 \%(\mathrm{r}=$ -0.67 to -0.71$)$ for IAT and between $53 \%$ and $59 \%(\mathrm{r}=$ -0.73 to -0.77 ) for PPO. Besides the traditional variables

Table 2 Results of the multiple regressions of race time 3 and z-time (standard error of the estimate (SEE), estimate, SD in seconds)

\begin{tabular}{|c|c|c|c|c|c|c|}
\hline & Step & $r^{2}$ (adjusted) & $\mathbf{P}$ & Estimate & SD & $\begin{array}{l}\text { Standard } \\
\text { beta }\end{array}$ \\
\hline $\begin{array}{l}\text { Race time } 3 \\
n=25\end{array}$ & $\begin{array}{l}\text { Intercept } \\
1 \text { min maximal effort body weight } \\
\text { IAT }\end{array}$ & $\begin{array}{l}0.868^{*} \\
\text { SEE: } 373.3\end{array}$ & $\begin{array}{r}<0.0001 \\
<0.0001 \\
<0.0001 \\
0.0002\end{array}$ & $\begin{array}{r}14132.362 \\
-10.045 \\
73.014 \\
-14.330\end{array}$ & $\begin{array}{c}611.5754 \\
14.614 \\
3.136 \\
1.434\end{array}$ & $\begin{array}{r}-0.882 \\
0.635 \\
-0.582\end{array}$ \\
\hline $\begin{array}{l}\text { z-time } \\
n=49\end{array}$ & $\begin{array}{l}\text { Intercept } \\
1 \text { min maximal effort body weight } \\
\text { IAT }\end{array}$ & $\begin{array}{l}0.757 \dagger \\
\text { SEE: } 0.48\end{array}$ & $\begin{array}{r}<0.0001 \\
<0.0001 \\
<0.0001 \\
0.0008\end{array}$ & $\begin{array}{r}2.356 \\
-0.009 \\
0.055 \\
-0.011\end{array}$ & $\begin{array}{l}0.499 \\
0.001 \\
0.009 \\
0.003\end{array}$ & $\begin{array}{r}-0.783 \\
0.581 \\
-0.460\end{array}$ \\
\hline
\end{tabular}

*With leaving-one-out cross-validation, $r^{2}$ was 0.835 .

†With leaving-one-out cross-validation, $r^{2}$ was 0.641 .

IAT, individual anaerobic threshold. 
of an incremental test, this study also recorded the data of an athlete's maximal average power output in three maximal efforts with various loading times $(10 \mathrm{~s}, 1 \mathrm{~min}$ and $5 \mathrm{~min}$ ). Interval durations and high-intensity intermittent loads are typical of MTB. ${ }^{1415}$ The power output in the 1 min maximal effort outperforms the predictive ability of the traditional incremental test in all three races. Very large correlations with race times 1,2 and 3 explaining between $68 \%$ and $72 \%(r=-0.82$ to -0.85$)$ of the race time variance were found. The $10 \mathrm{~s}$ all-out sprint showed inferior results. The predictive ability of the $5 \mathrm{~min}$ maximal effort was similar to the traditional parameters, but in the multiple regression model the 5 min maximal effort did not explain any further variance of the performance, indicating that the $5 \mathrm{~min}$ maximal effort could be excluded in the future to abbreviate the test protocol. The use of the $1 \mathrm{~min}$ maximal effort $(1 \mathrm{~min})$ and the traditional incremental test parameter IAT in a multiple regression model confirmed additional explanatory power to predict XCM performance $(87 \%$ of race time 3 and $76 \%$ of z-time).

\section{Comparison with previous studies}

The traditional laboratory variables (scaled by body weight $(\mathrm{W} / \mathrm{kg})$ ) were also analysed by other studies for the mountain bike discipline cross-country. A comparable correlation $(\mathrm{r}=-0.64)$ between race time and the athlete's onset of lactate threshold ${ }^{28}$ was described by Prins $e t a l^{12}$ in eight elite mountain bikers. The relation between race time and the lactate threshold ${ }^{29}$ reported by Impellizzeri $e t a l,{ }^{13}$ who analysed 13 national-level athletes, was stronger $(r=-0.86)$. Costa and De-Oliveira ${ }^{6}$ analysed six national-level athletes and found different correlations between lactate threshold ${ }^{30}$ and final race rank position in two XCO races $(\mathrm{r}=-0.32$ and $\mathrm{r}=-0.78)$. Impellizzeri $e t$ $a l^{11}$ analysed 12 international-level athletes and reported correlations between $\mathrm{PPO}$ and race time of $\mathrm{r}=-0.48$. Costa and De-Oliveira ${ }^{6}$ found a very large correlation between PPO and final race rank position of two races $(\mathrm{r}=-0.88$ and $r=-0.88)$. The traditional parameters of the incremental test showed large variability between the studies, indicating the difficulty to predict race performance in MTB.

To address this, we combined traditional parameters of an incremental test and maximal efforts. In this regard, the incremental test at the beginning of the performance test influences the power output of the maximal efforts because athletes cannot completely recover during the recovery periods. Because of increasing fatigue during the performance test, the power outputs of the maximal efforts are expected to be smaller than those seen without preloading. Moreover, the power outputs of the maximal efforts might be influenced by familiarisation and experience with these tests.

Despite these limitations of comparing the predictive ability of the power outputs of the maximal efforts with those seen with other tests that focus on short-term and mid-term intervals, the predictive ability of the power outputs obtained in this study will now be compared with those of previously published studies on XCO. They also tried to improve the prediction of race performance by using alternative testing methods. Costa and De-Oliviera $^{6}$ only found small and non-significant correlations between mean power output scaled by body weight over $30 \mathrm{~s}$ and the final race rank position in two XCO races $(\mathrm{r}=-0.12 ; \mathrm{r}=-0.29)$. Inoue $e t a t^{2}$ tested $10 \mathrm{XCO}$ riders who performed five repeated Wingate tests with 30 s recovery between the Wingate tests. The average power output in all five Wingate tests scaled by body weight $(\mathrm{r}=-0.63$; $\mathrm{P}<0.05)$ and the peak power of all five Wingate tests scaled by body weight $(\mathrm{r}=-0.79 ; \mathrm{P}<0.01)$ correlated significantly with race time. Miller $e t a l^{14}$ used a field-based test to predict the XCO performance of 11 regionally competitive athletes. They reported a large correlation between race time and intermittent power scaled by body weight of 20 intervals of $45 \mathrm{~s}$ work and $15 \mathrm{~s}$ recovery $(\mathrm{r}=0.89$; $\mathrm{P}<0.001)$. This correlation is similar to those observed between power output in the 1 min maximal effort and race times in this study. However, smaller correlations were found by Prins $e t a l^{12}$ between performance times for $1 \mathrm{~km}$ time trials and race time. Participants needed $80-95 \mathrm{~s}$ to complete the $1 \mathrm{~km}$ time trial. Therefore, durations were similar to those of the $1 \mathrm{~min}$ maximal effort in this study. Each participant $(n=8)$ performed a $1 \mathrm{~km}$ time trial from rest $(\mathrm{r}=0.29)$ after a $26 \mathrm{~min}(\mathrm{r}=0.53)$ and a $52 \mathrm{~min}(\mathrm{r}=0.59)$ laboratory test with variable fixed intensities to simulate an $\mathrm{XCO}$ race.

\section{Six considerations when comparing with previous studies}

A comparison of our findings with those of other studies may by hampered by several factors: (1) As described by Impellizzeri et $a l^{11}$ small variations in correlation coefficients between studies might be explained by differences in the distinct sample size. The variability of the race times in the studies by Prins $e t a l,{ }^{12}$ Impellizzeri et $a l^{13}$ and Impellizzeri et $a l^{11}$ was similar and less pronounced than that of the race times in this study, indicating more homogeneous study populations in the previous investigations. Other studies did not report the ranges of the dependent variable. (2) In addition, the small sample sizes in the previous studies resulted in large CIs. For example, Prins $e t a l^{12}$ mentioned that the low correlations of the $1 \mathrm{~km}$ trials could have been the result of a type II error. (3) Moreover, athletes' performance levels in this study, which were measured with the absolute PPO as suggested by De Pauw et al, ${ }^{21}$ were lower than those of international competitive XCO athletes in the study by Impellizzeri $e t$ $a l^{11}(\mathrm{PPO}=426 \mathrm{~W})$ but higher than those of XCM athletes in the study by Wirnitzer and Kornexl. ${ }^{7}$ (4) In addition, different calculation methods of lactate threshold and (5) differences in the incremental test protocol could influence the correlations found in the different studies. Moreover, previous reports compared predictors with the (6) race performance in XCO races. These races are more intense and shorter in duration than XCM races. ${ }^{47}$ In reference to the popularity of XCM as a recreational 
and competitive sport, this study can therefore fill a diagnostic gap by allowing recommendations for XCM performance.

However, different race characteristics between XCM races also influence the correlations. In this study, correlation coefficients between race times and the afforded power in the $10 \mathrm{~s}$ all-out sprint and $5 \mathrm{~min}$ maximal effort showed variation between the races. IAT, PPO and the 1 min maximal effort are less dependent on race characteristics because the correlations found were constant over all three races. Even the fact that race 2 was a stage race does not influence the explanatory power of the results. The generalisability of IAT, PPO and $1 \mathrm{~min}$ maximal effort is therefore better than that of power in the $10 \mathrm{~s}$ all-out sprint and in the $5 \mathrm{~min}$ maximal effort.

The aforementioned studies have investigated the correlations between physiological-based measures and the endurance performance of only one $e^{211-14}$ and two ${ }^{6}$ races. The results of our study could demonstrate that the results of a single comparison between laboratory variables and a specific race performance in XCM overestimate the predictive value of variables when considering different races. This can be seen in the lower correlation coefficients between z-time and laboratory variables compared with correlation coefficients of the single races.

\section{Multiple regression models}

Due to the large sample size, multiple regression models for a more precise race prediction could be calculated with three predictors. Using backward calculation for race time 3 as well as the z-transformed race time, two significant models could be calculated with the same predictor variables. This new test protocol was able to explain up to $86.8 \%$ of the variance of a single XCM race. This value is slightly smaller than the explanatory power described for laboratory tests to predict a $10 \mathrm{~km}$ run $\left(r^{2}=0.889\right)$, half marathon $\left(r^{2}=0.924\right)$ and marathon $\left(r^{2}=0.899\right)$ with three independent variables. ${ }^{31}$ In contrast to a running marathon with a predefined distance of $42.2 \mathrm{~km}$ and no relevant differences in altitude or challenging running surfaces, XCM races differ remarkably with respect to length, altitude and technical demands. Those race characteristics could have been added to the calculation model. However, more validation races would have been necessary to obtain a valid model. Therefore, the calculated models between predictor variables and response variables are not as generalisable as the results of a laboratory test to predict the finish time of a running marathon. Calculating data across all three races reduced the explanation of variance for all three race times $\left(r^{2}=0.76\right)$. Consequently, the inclusion of different races into one model via z-transformed data results in a lower explanatory power but a higher external validity of the model.

\section{Practical application and further research}

This study verified the test protocol's validity for trained, amateur XCM athletes. The additional explanatory power of the comprehensive protocol is possible through the analysis of aerobic variables and maximal efforts. In total, this protocol takes approximately $50 \mathrm{~min}$, including warm-up and cool-down periods. This laboratory-based performance test can therefore be implemented into a clinical routine. However, this laboratory-based performance test is limited by smaller ecological validity compared with field-based performance tests. ${ }^{32}$

Apart from predicting race performance, this protocol can be used to create individual athlete profiles with regard to different physiological demands of XCM. An athlete's profile can be compared with the results of other athletes, and individual strengths and weaknesses can be outlined and implemented into recommendations for training. In this regard, repeated tests allow a detailed control of the training process and are therefore valuable for athletes and coaches.

Further research is needed to evaluate the test reliability and training recommendations based on the results of the test. Moreover, the validity of the performance test and the study's results should be analysed in further studies for the XCO discipline and for professional cyclists.

\section{CONCLUSION}

All laboratory variables of the tested study protocol correlated at least largely with the athletes' compulsory times for singular XCM races. The new approach with an incremental bicycle ergometer test to determine IAT and PPO and the additional maximal efforts with durations of $10 \mathrm{~s}, 1 \mathrm{~min}$ and $5 \mathrm{~min}$ represents a valid performance test to predict XCM race performance. In this regard, the power output of the 1 min maximal effort, representing the intensive demands of an XCM race, is the most valuable single variable. IAT, PPO and the 1 min maximal effort have shown themselves to be stable predictors in a multivariate approach.

Acknowledgements We acknowledge support by Deutsche Forschungsgemeinschaft and Open Access Publishing Fund of University of Tübingen. The authors would like to thank the race organisations that supported the recruitment of athletes by announcing the study on their call for race or website.

Contributors The manuscript has been read and approved by all the listed coauthors. M-DA: conception and design of the study, data acquisition, analysis and interpretation of data, drafting the manuscript, final approval. PS: conception and design of the study, interpretation of data, final approval. PM: analysis and interpretation of data, statistics, revising the article, final approval. AMN: supervision, design of the study, revising the article, final approval. IK: conception and design of the study, interpretation of data, drafting the manuscript, final approval.

Funding This research received no specific grant from any funding agency in the public, commercial or not-for-profit sectors.

Competing interests None declared.

Patient consent Obtained.

Ethics approval The study was approved by the local ethics committee of the University Clinic of Tuebingen (229/2013/B02) according to the Declaration of Helsinki.

Provenance and peer review Not commissioned; externally peer reviewed.

Data sharing statement No further unpublished data are available. 
Open Access This is an Open Access article distributed in accordance with the Creative Commons Attribution Non Commercial (CC BY-NC 4.0) license, which permits others to distribute, remix, adapt, build upon this work non-commercially, and license their derivative works on different terms, provided the original work is properly cited and the use is non-commercial. See: http://creativecommons.org/ licenses/by-nc/4.0/

(c) Article author(s) (or their employer(s) unless otherwise stated in the text of the article) 2018. All rights reserved. No commercial use is permitted unless otherwise expressly granted.

\section{REFERENCES}

1. Stapelfeldt B, Schwirtz A, Schumacher YO, et al. Workload demands in mountain bike racing. Int J Sports Med 2004;25:294-300.

2. Inoue A, Sá Filho AS, Mello FC, et al. Relationship between anaerobic cycling tests and mountain bike cross-country performance. J Strength Cond Res 2012;26:1589-93.

3. Baron R. Aerobic and anaerobic power characteristics of off-road cyclists. Med Sci Sports Exerc 2001;33:1387-93.

4. Impellizzeri FM, Marcora SM. The physiology of mountain biking. Sports Med 2007;37:59-71

5. Union Cycliste Internationale. Uci cycling regulations Part IV. Mountain bike races. 2016. http://www.uci.ch/mm/Document/News/ Rulesandregulation/17/29/73/4MTB-E-1.01.2016_English.pdf

6. Costa V, De-Oliveira F. Physiological variables to predict performance in cross-country mountain bike races. J Exerc Physiol Online 2008;11:14-24.

7. Wirnitzer KC, Kornexl E. Exercise intensity during an 8-day mountain bike marathon race. Eur J Appl Physiol 2008;104:999-1005.

8. Miller MC, Witmer C, Moir GL, et al. Predictive Validity of Critical Power and Functional Threshold Power for Mountain Bike Race Performance. Int J Exerc Sci: Conference Proceedings 2014;9:55.

9. Stevens CJ, Dascombe BJ. The reliability and validity of protocols for the assessment of endurance sports performance: an updated review. Meas Phys Educ Exerc Sci 2015;19:177-85.

10. Currell K, Jeukendrup AE. Validity, reliability and sensitivity of measures of sporting performance. Sports Med 2008;38:297-316.

11. Impellizzeri FM, Marcora SM, Rampinini E, et al. Correlations between physiological variables and performance in high level cross country off road cyclists. Br J Sports Med 2005;39:747-51.

12. Prins $\mathrm{L}$, Terblanche E, Myburgh KH. Field and laboratory correlates of performance in competitive cross-country mountain bikers. $J$ Sports Sci 2007;25:927-35.

13. Impellizzeri FM, Rampinini E, Sassi A, et al. Physiological correlates to off-road cycling performance. J Sports Sci 2005;23:41-7.

14. Miller MC, Moir GL, Stannard SR. Validity of using functional threshold power and intermittent power to predict cross-country mountain bike race outcome. Journal of Science and Cycling 2014;3-16-20.
15. Allen $\mathrm{H}$, Coggan A. Training and racing with a power meter. Boulder, Colorado: Velo Press, 2010

16. Impellizzeri FM, Ebert T, Sassi A, et al. Level ground and uphill cycling ability in elite female mountain bikers and road cyclists. Eur $J$ Appl Physiol 2008;102:335-41.

17. Impellizzeri F, Sassi A, Rodriguez-Alonso $M$, et al. Exercise intensity during off-road cycling competitions. Med Sci Sports Exerc 2002;34:1808-13.

18. Novak AR, Dascombe BJ. Physiological and performance characteristics of road, mountain bike and BMX cyclists. Journal of Science and Cycling 2014;3:9-16.

19. Lee H, Martin DT, Anson JM, et al. Physiological characteristics of successful mountain bikers and professional road cyclists. J Sports Sci 2002;20:1001-8.

20. Ahrend M, Schneeweiss $\mathrm{P}$, Comparison of laboratory parameters of a mountain bike specific performance test and a simulated race performance in the field. Journal of Science and Cycling 2016;5:3-9.

21. De Pauw K, Roelands B, Cheung SS, et al. Guidelines to classify subject groups in sport-science research. Int J Sports Physiol Perform 2013;8:111-22.

22. Dickhuth HH, Yin L, Niess A, et al. Ventilatory, lactate-derived and catecholamine thresholds during incremental treadmill running: relationship and reproducibility. Int J Sports Med 1999;20:122-7.

23. Roecker K, Striegel H, Dickhuth $\mathrm{HH}$. Heart-rate recommendations: transfer between running and cycling exercise? Int J Sports Med 2003;24:173-8

24. Fernández-Peña $E$, Lucertini $F$, Ditroilo $M$. A maximal isokinetic pedalling exercise for EMG normalization in cycling. J Electromyogr Kinesiol 2009;19:e162-170.

25. Macdermid PW, Morton RH. A longitudinal analysis of start position and the outcome of World Cup cross-country mountain bike racing. J Sports Sci 2012;30:175-82.

26. Swain DP. The influence of body mass in endurance bicycling. Med Sci Sports Exerc 1994;26:58???63-63.

27. Hopkins WG. A new view of statistics. http://sportsci.org/resource/ stats/1997 (accessed 1 Feb 2016).

28. Sjödin B, Jacobs I. Onset of blood lactate accumulation and marathon running performance. Int J Sports Med 1981;2:23-6.

29. Hagberg JM, Coyle EF. Physiological determinants of endurance performance as studied in competitive racewalkers. Med Sci Sports Exerc 1983;15:287-9.

30. Berg A, Jakob E, Lehmann M, et al. Current aspects of modern ergometry. Pneumologie 1990;44:2-13.

31. Roecker K, Schotte O, Niess AM, et al. Predicting competition performance in long-distance running by means of a treadmill test. Med Sci Sports Exerc 1998:30:1552-7.

32. Jobson SA, Nevill AM, Palmer GS, et al. The ecological validity of laboratory cycling: Does body size explain the difference between laboratory- and field-based cycling performance? J Sports Sci 2007;25:3-9. 